\title{
Improvement of implicit finite element code performance in deep drawing simulations by dynamics contributions
}

\author{
T. Meinders * , A.H. van den Boogaard, J. Huétink \\ University of Twente, Department of Mechanical Engineering, P.O. Box 217, 7500 AE \\ Enschede, The Netherlands
}

\begin{abstract}
To intensify the use of implicit finite element codes for solving large scale problems, the computation time of these codes has to be decreased drastically. A method is developed which decreases the computational time of implicit codes by factors. The method is based on introducing inertia effects into the implicit finite element code in combination with the use of iterative solvers. Another advantage of introducing inertia effects into an implicit finite element code is that it stabilizes the computation, especially when the problem is under-constrained. The dynamics contributions are successfully implemented for both the plane strain element (only displacement d.o.f.) and the Mindlin shell element (displacement and rotational d.o.f.). Deep drawing simulations are performed to investigate the performance of the dynamics contributions in combination with iterative solvers. It is concluded that the computation time can be decreased by a factor 5-10.
\end{abstract}

Key words: dynamics contributions, implicit finite element code, sheet forming process

\section{Introduction}

The two main solution procedures for the simulation of deep drawing processes are the dynamic explicit and the static implicit procedure. The dynamic explicit method is frequently used in simulations of the deep drawing process, since it reduces the computational time drastically, using a diagonal mass matrix system to solve the equations of motion [1]. For this method, the computational time depends linearly on the number of degrees of freedom (d.o.f.). However, the drawbacks of the explicit method are that small time steps are needed and that equilibrium after each

\footnotetext{
* Corresponding author.

Email address: v.t.meindersectw. utwente.nl (T. Meinders).
} 
time step cannot be checked, which can result in wrong stress distributions and unrealistic product shapes [2]. Another drawback is that springback calculations are very time consuming. The advantage of a static implicit method is that equilibrium is checked after each time step and thus will lead to more accurate results. The main drawback of this method however, is that the computation time depends quadratically on the number of d.o.f. if a direct solver is used. Another drawback is that the stiffness matrix is often ill conditioned which can make the method unstable and detoriates the performance of iterative solvers.

At the present time the competition between implicit and explicit finite element codes is still in full swing, where explicit codes are favored for solving large problems although implicit codes yield more accurate results. To intensify the use of implicit codes for solving large problems, the computation time of these codes has to be decreased drastically. A method is developed which decreases the computational time of implicit codes by factors, which makes the implicit finite element code competitive with explicit finite element code for large scale problems. This method is based on introducing dynamics contributions into the implicit finite element code. As a result, a mass matrix is introduced. When this mass matrix is diagonalized and added to the stiffness matrix, the diagonal terms of the system matrix will increase and the matrix will be better conditioned, which makes an effective use of iterative solvers possible. Another advantage of introducing dynamics contributions into an implicit finite element code is that it stabilizes the computation, especially when the problem is under-constrained. For example, when closing a doubly curved blankholder, the sheet only makes contact at two or three points which give rise to stability problems when the simulation is started and no inertia effects are present in the simulation.

This article starts with the basics of the implementation of the dynamics contributions into the implicit finite element code DiekA, Section 2. In sheet metal forming, shell elements are used which have three displacement d.o.f. and three rotational d.o.f. per node. These rotational d.o.f. give rise to complications in the implementation of dynamics contributions. Therefore we start with the implementation of dynamics contributions in the two-dimensional plane strain element, which has only displacement d.o.f., Section 3. Then the implementation is proceeded for shell elements, Section 4. In Section 5 the performance of the dynamics contributions in combination with the use of iterative solvers is investigated for a simple deep drawing simulation. Section 6 shows that the dynamics contributions stabilizes an under-constrained problem. In Section 7 the results of the simulation of a front door panel are discussed [3]. The article is closed with some concluding remarks and a recommendation. 


\section{Dynamics contributions}

Omitting damping influences, the discretized equations of motion in the linear case read:

$$
M \ddot{u}_{n}+K u_{n}=F_{n}
$$

For non-linear computations the second term of the left hand side is replaced by the internal force vector. Two integration methods to calculate the displacements and velocities will be compared, Section 2.1. Then a consistent or a lumped mass matrix can be derived, Section 2.2.

\subsection{Integration Methods}

Using the backward difference method, Equation 1 can be written in incremental form:

$$
\left(\frac{M}{\Delta t^{2}}+K\right) \delta \Delta u_{n}^{k}=F_{n}-R_{n}^{k}-M \ddot{u}_{n}^{k}
$$

Note that the Backward difference scheme suffers from numerical damping for relatively large time increments.

Using the Newmark integration method, Equation 1 reads in incremental form:

$$
\left(\frac{M}{\beta \Delta t^{2}}+K\right) \delta \Delta u_{n}^{k}=F_{n}-R_{n}^{k}-\frac{M}{\beta \Delta t^{2}} \Delta u_{n}^{k}+\frac{M}{\beta \Delta t} \dot{u}_{n-1}+\left(\frac{1}{2 \beta}-1\right) M \ddot{u}_{n-1}
$$

where the parameters $\delta$ and $\beta$ are free to choose. In the linear case, The Newmark integration method is unconditionally stable for $\delta=0.5$ and $\beta=0.25$. However, the scheme may lose stability in the nonlinear case [4].

\subsection{Mass Matrix}

The consistent mass matrix can be calculated through the spatial discretization of the weak form of equilibrium:

$$
\rho \int_{V}(\delta \dot{u}) \ddot{u} d V=\rho \sum_{\alpha} \sum_{\beta} \int_{V} \delta u^{\alpha} N^{\alpha} N^{\beta} \ddot{u}^{\beta} d V=\delta u^{\alpha} \rho \int_{V} N^{\alpha} N^{\beta} d V \ddot{u}^{\beta}=\delta \dot{u}^{\alpha} M^{\alpha \beta} \ddot{u}^{\beta}
$$

In case of using a lumped mass matrix, the mass matrix is diagonalized. This can simply be done by adding all terms in a row and put it on the diagonal. The lumped mass matrix method is frequently used in explicit codes. The main advantage of this 
method is that the inverse of this matrix is easily calculated by taking the inverse of each diagonal term. A second advantage of the lumped mass matrix is that it will increase the condition number of the system matrix.

\section{Implementation of dynamics contributions for plane strain elements}

\subsection{One element test}

To validate the dynamics contributions, a one element test is used to calculate the oscillation time and the maximum amplitude of a mass-spring system, see Figure 1. The element width is $1 \mathrm{~mm}$, the element length is $1 \mathrm{~mm}$, the density $\rho$ is 0.001 tons $/ \mathrm{mm}^{3}$, the Poisson ratio $v$ is 0 and the Young's modulus $E$ is $100 \mathrm{~N} / \mathrm{mm}^{2}$. Note that the density is given in tons $/ \mathrm{mm}^{3}$ to have a consistent set of units.

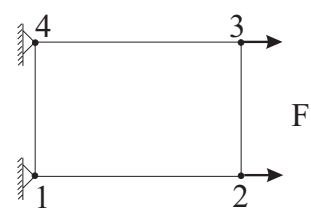

Fig. 1. plane strain element with boundary conditions.

While using the lumped mass matrix, the one element test will degenerate to a mass-spring system, where the mass is concentrated in the nodes and the springs will be the stiffness of the element. Since nodes 1 and 4 are fully suppressed, the mass will not be taken into account in these two nodes which means that nodes 2 and 3 contain half of the total mass. Nodes 2 and 3 are loaded with a unit force. The analytical oscillation time for this one element test is [5]:

$$
t=\frac{2 \pi}{\sqrt{\frac{A E}{L}}} \sqrt{m}=\frac{2 \pi}{\sqrt{100}} \sqrt{0.0005}=0.0140 \mathrm{~s}
$$

The simulated oscillation time is $\mathrm{t}=0.0139$, which perfectly matches the analytical solution.

The analytical response of the mass-spring system on the step force load reads [6]:

$$
x(t)=\frac{F}{k}(1-\cos (\omega t-\phi))
$$

The maximum response for this particular situation gives:

$$
x_{\max }(t)=\frac{2 F}{k}=\frac{2 \cdot 2}{100}=0.04 \mathrm{~mm}
$$


The simulated maximum amplitude is $0.0419 \mathrm{~mm}$, which shows a good agreement with the analytical solution, where the slight deviation between the analytical and the simulated result can be subscribed to geometrical non-linearities.

\subsection{Beam bending test}

The beam bending test, see Figure 2, is performed to check whether the beam will oscillate when realistic values are used in a simulation. The beam length is $50 \mathrm{~mm}$ and is modeled with 20 elements. The density $\rho$ is $7.8 \cdot 10^{-9}$ tons $/ \mathrm{mm}^{3}$, the Poisson ratio $v$ is 0 and the Young's modulus $E$ is $210000 \mathrm{~N} / \mathrm{mm}^{2}$.

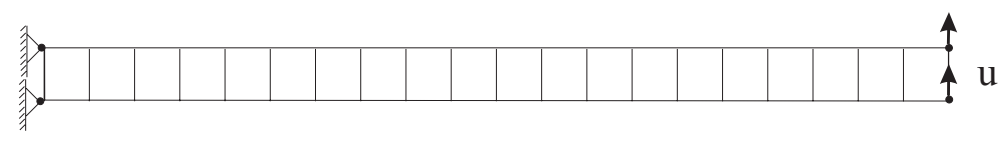

Fig. 2. Beam modeled with plane strain elements.

Two simulations are performed in which a certain displacement is prescribed where after the beam is released, using the backward difference scheme and the Newmark integration scheme, respectively. The lumped mass matrix approach is used. The results of these simulation are given in Figure 3.

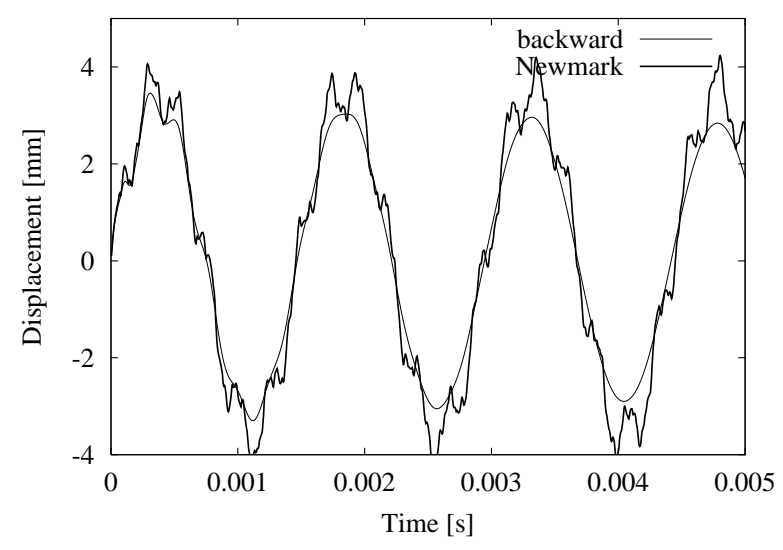

Fig. 3. Oscillation for beam modeled with plane strain elements.

Figure 3 clearly shows that several natural frequencies of the beam are activated, in case the Newmark difference scheme is applied. In other words, the first natural frequency of the beam is superimposed by higher order frequencies. Note that, in case the Backward difference scheme is applied, the higher order terms will damp more, and as a result the beam will further vibrate in its first natural frequency. The 
analytical solutions for the natural frequencies of the beam are [7]:

$$
f_{n}=\frac{C_{n}}{2 \pi} \sqrt{\frac{E I}{\rho A l^{4}}}
$$

with $C_{1}=3.52, C_{2}=22.4, C_{3}=61.7$ and $C_{4}=121.0$. The first natural frequency is $335.2 \mathrm{~Hz}$ for this beam (analytical). However, the simulation gives a first natural frequency of $676.7 \mathrm{~Hz}$. The deviation between these values is due to the fact that the plane strain element acts too stiff for bending modes when the element size is not small enough (caused by an overestimation of the shear stress). For the given geometry, the overestimation of the stiffness of the beam is a factor 4.124 (analytical deflection versus simulated deflection). Consequently, the simulated natural frequencies will be a factor 2.03 to high. Modification of the simulated natural frequencies with this factor gives:

\begin{tabular}{|l|r|r|}
\hline frequency & analytical $[\mathrm{Hz}]$ & simulation (modified) $[\mathrm{Hz}]$ \\
\hline$f_{1}$ & 335.2 & 333.2 \\
$f_{2}$ & 2133.5 & 2085.0 \\
$f_{3}$ & 5875.6 & 5755.3 \\
$f_{4}$ & 11522.6 & 11093.2 \\
\hline
\end{tabular}

Note that the results are less accurate for increasing frequency, since the wavelength will be shorter and as a result more difficult to describe with only 20 elements.

From these results, it can be concluded that the natural frequencies are simulated sufficiently accurate. The previous section proved that amplitude and oscillation time can be accurately determined, using the dynamics contributions for plane strain elements. Consequently, the implementation of the dynamics contributions is proceeded for shell elements.

\section{Implementation of dynamics contributions for shell elements}

Three approaches can be considered to determine the mass contributions for both the displacement and rotational d.o.f. for a 3-node shell element. The first approach only takes into account the lumped mass contribution to the displacement d.o.f. The second approach makes use of a consistent mass matrix, derived by Hermitian polynomials. The third approach uses mass moments of inertia to take into account the mass contributions for the rotational d.o.f.

This section will focus on the beam bending test, since the simulations of the one element test gave identical results to the plane strain elements (Section 3.1). How- 
ever, different results are expected for the beam bending test since, contrary to the used plane strain element, Mindlin shell elements can accurately describe bending modes [8]. In Section 4.1, the different approaches will be explained in detail and compared with each other. Subsequently one of the approaches is chosen to be used, where after this section continues, using the chosen approach.

\subsection{Three approaches to implement the dynamics contributions}

\subsubsection{Approach 1: Only displacement d.o.f.}

Beforehand, it is not clear what the influence will be of taking into account mass contributions for the rotational d.o.f. Therefore only the displacement d.o.f. are focused on. Using the lumped mass matrix approach and the evaluation of area integrals according to [9], the mass matrix for a linear triangular element can be written as:

$$
M=\left[\begin{array}{ccccc}
\frac{1}{3} & 0 & 0 & 0 & \ldots \\
0 & \frac{1}{3} & 0 & 0 & \ldots \\
0 & 0 & \frac{1}{3} & 0 & \ldots \\
0 & 0 & 0 & 0 & \ldots \\
\vdots & \vdots & \vdots & \vdots & \ddots
\end{array}\right] \rho h A
$$

with $h$ the thickness of the element. The first 3 d.o.f. represent the translations of the first node, the following 3 d.o.f. represent the rotations of the first node. Subsequently, node 2 and 3 follow.

\subsubsection{Approach 2: Hermitian polynomials}

The rotational d.o.f. influence the displacement normal to the element plane. Therefore a coupling between these d.o.f. has to be generated through the interpolation functions. A neat coupling method is based on Hermitian polynomials [10], which yields a consistent mass matrix. However, lumping this consistent mass matrix gives negative values on the diagonal of the lumped mass matrix which is very bad for the condition number of the mass matrix. 


\subsubsection{Approach 3: Mass moment of inertia}

Another method to implement mass contributions to the rotational d.o.f. is based on mass moments of inertia:

$$
\delta W=\delta \omega I_{m} \ddot{\omega} \approx \delta \omega \rho h I \ddot{\omega} \approx \delta \omega \rho h\left(\frac{1}{3} A^{2}\right) \ddot{\omega}
$$

with $A$ the element area. In case of linear triangular elements, this approach yields the following lumped mass matrix:

$$
M=\left[\begin{array}{ccccc}
\frac{1}{3} & 0 & 0 & 0 & \ldots \\
0 & \frac{1}{3} & 0 & 0 & \ldots \\
0 & 0 & \frac{1}{3} & 0 & \ldots \\
0 & 0 & 0 & \frac{1}{9} A & \ldots \\
\vdots & \vdots & \vdots & \vdots & \ddots
\end{array}\right] \rho h A
$$

\subsubsection{Comparison of the three approaches}

The selection criterion to choose the right approach will be which one yields the highest increase of the condition number of the system matrix while preserving an accurate solution. From this point of view, approach 2 can already be dropped. The remaining two approaches are compared, using the beam bending test. The results showed that only a slight difference in amplitude was observed when mass is added to the rotational d.o.f. Based on these results and looking at the usage of iterative solvers, the mass moment of inertia approach is preferred, since this approach yields the highest increase of the condition number of the system matrix.

\subsection{Beam bending test}

Two simulations are performed, using the Newmark and backward integration scheme, respectively, Figure 4. 


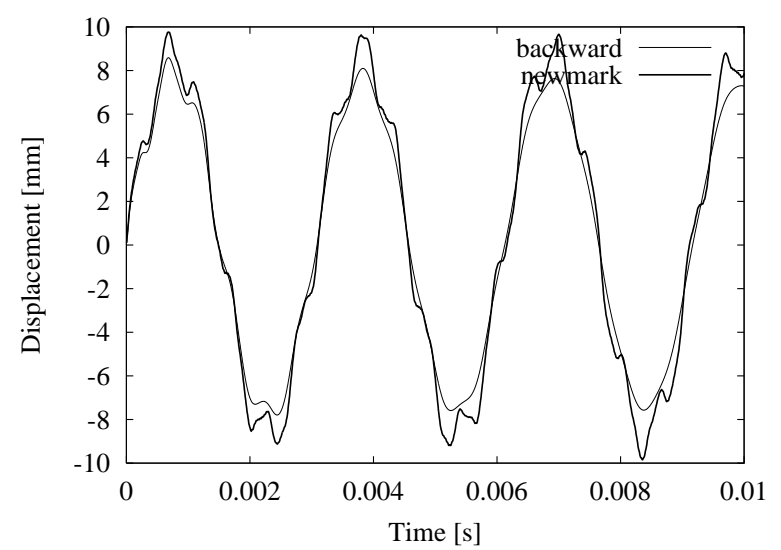

Fig. 4. Beam modeled with Mindlin elements.

It is clear that also the higher order frequencies are spotted when Mindlin elements are used. The first natural frequency, calculated with the Mindlin beam is 328.9 $\mathrm{Hz}$, which shows a good agreement with the analytical solution $(335.2 \mathrm{~Hz}$, see Section 3.2).

\section{Deep drawing of a rectangular product}

Section 4 showed that the dynamics contributions for shell elements are correctly implemented in the implicit finite element code. In this section the influence of the dynamics contributions on the deep drawing of a rectangular product is investigated. The main goal of the dynamics contributions is to stabilize the calculation and not to be able to accurately describe dynamic effects. Therefore the backward difference scheme is used instead of the Newmark integration scheme, the numerical damping restrains the vibration modes. The lumped mass matrix approach is used to improve the condition number of the system stiffness matrix. Following the strategy used in explicit codes (artificial scaling of punch velocity to benefit from dynamics contributions), the punch velocity is set to $50 \mathrm{~m} / \mathrm{s}$. The initial dimensions of the plate are $313 \mathrm{~mm} \times 221 \mathrm{~mm}$. The plate thickness is $1 \mathrm{~mm}$ and the density $\rho$ is $7.8 \cdot 10^{-9}$ tons $/ \mathrm{mm}^{3}$. The product depth is $100 \mathrm{~mm}$.

One simulation is performed without dynamics contributions, and one simulation is performed with dynamics contributions. Some differences appear between both simulations. At the start of the 'dynamics' simulation, the displacement of the plate under the punch in z-direction is smaller than the displacement of the "no dynamics' simulation due to inertia effects. When the simulation proceeds, the 'dynamics' simulation shows a higher displacement in z-direction. However, after $50 \mathrm{~mm}$ some damping is observed which is due to plasticity and numerical damping. At the final stage $(100 \mathrm{~mm})$, almost no difference is observed in the z-displacements for both simulations. 
The implementation of the dynamics contributions also influences the convergence behavior of the Newton-Raphson procedure. The simulation without dynamics contributions needs 565 iterations for the entire simulation. The simulation, using the backward difference method, needs 455 iterations for the entire simulation. The convergence behavior of both simulations is graphically represented in Figure 5.

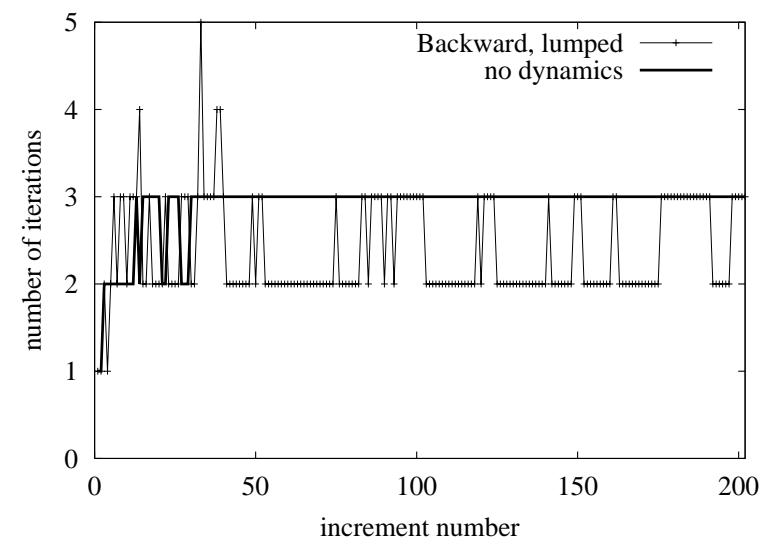

Fig. 5. Convergence behavior.

The thickness strain distribution in the product is compared for both simulations, see Figure 6 and Figure 7. It is observed that the dynamics contributions do influence the thickness strain distribution. However, note that this was also expected due to the high velocity of the simulated deep drawing process $(50 \mathrm{~m} / \mathrm{s})$. When a simulation is performed with a slower drawing speed $(25 \mathrm{~m} / \mathrm{s})$, hardly any difference is observed between the non-dynamic simulation and the simulation with dynamics contributions.

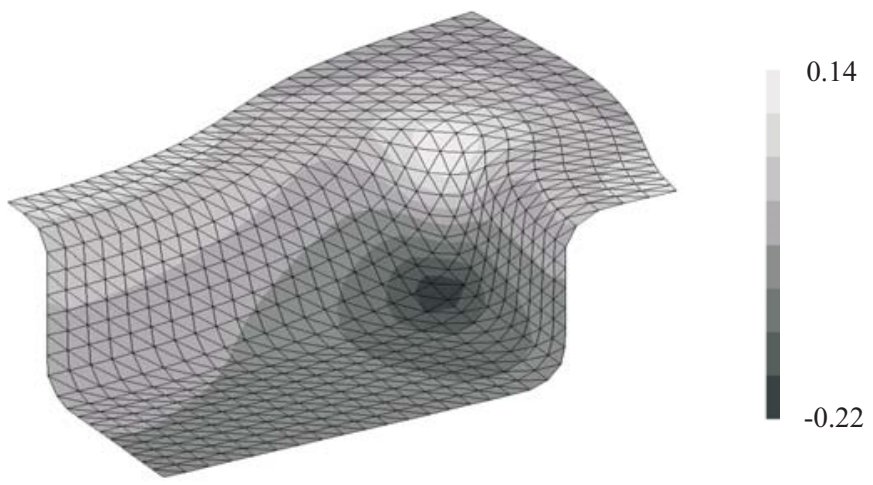

Fig. 6. Thickness strain distribution in product, without dynamics contributions. 


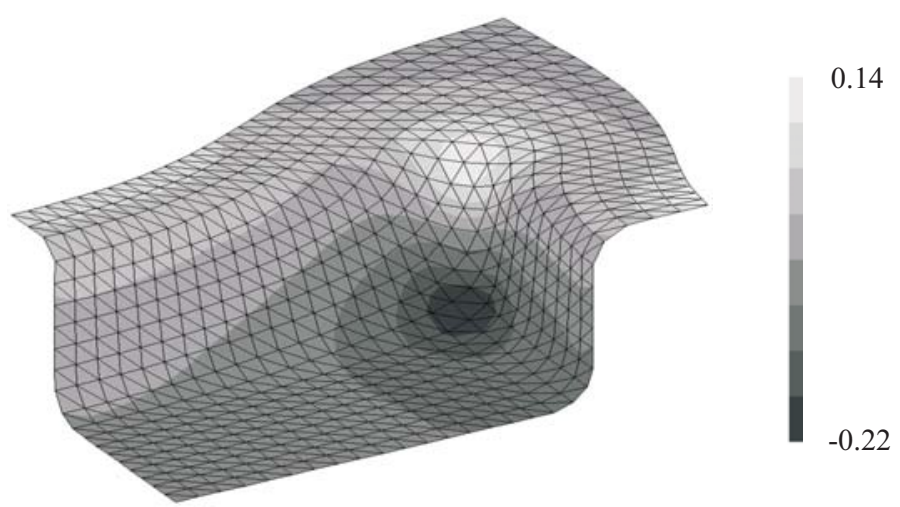

Fig. 7. Thickness strain distribution in product, using dynamics contributions.

All former simulations are performed, using a sparse matrix solver (Cholesky decomposition). Since it is expected that the dynamics contributions will improve the condition of the matrix (and thus improve the convergence behavior of iterative solvers), the convergence behavior of different iterative solvers is investigated. The iterative solvers used are the Conjugate Gradient (CG) solver, the Generalized Minimum Residual (GMRES) solver and the Biconjugate Gradient Stabilized (BiCGSTAB) solver, all in combination with a Symmetric Successive Over Relaxation (SSOR) preconditioner [11]. Several simulations are performed in which the punch velocity is varied between $0 \mathrm{~m} / \mathrm{s}$ (no dynamics contributions) and $50 \mathrm{~m} / \mathrm{s}$. The system matrix at step 40 is dumped where after the system is solved, using the different iterative solvers. The unbalance criterion is set to $10^{-5}$. The performance of these iterative solvers is given in Figure 8. It can be concluded that the convergence rate drastically increases with an increase of the deep drawing velocity. Since, the results between a simulation performed with dynamics contributions until a deep drawing speed of $25 \mathrm{~m} / \mathrm{s}$ and one without dynamics contributions do not differ significantly, it is possible to decrease the computation time by factors without affecting the results. In case of the GMRES solver the computation time is reduced by more than a factor 12, for the CG solver with more than a factor 6 and for the Bi-CGSTAB solver with more than a factor 2 .

Finally, a set of simulations is performed without dynamics contributions for the rotational d.o.f. The results of these simulations are shown in Figure 9. This figure shows that it is important to take into account the dynamics contributions for the rotational d.o.f., since they have a large influence on the convergence behavior. 


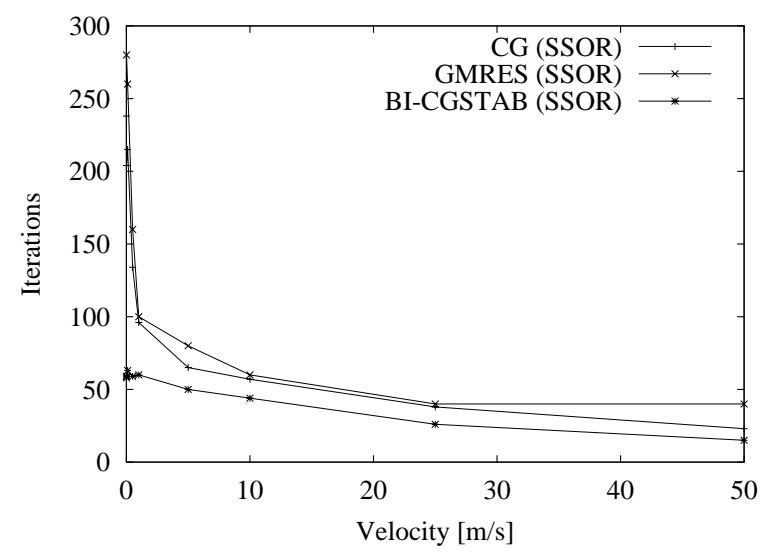

Fig. 8. Convergence behavior of different iterative solvers, dynamics contributions on displacement and rotational d.o.f.

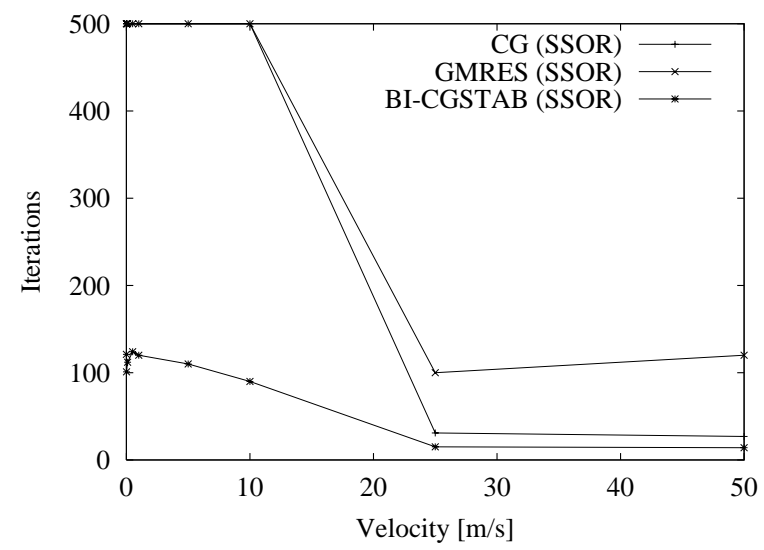

Fig. 9. Convergence behavior of different iterative solvers, dynamics contributions on displacement d.o.f. only.

\section{Stabilization of under-constrained problem}

The dynamics contributions stabilize the computation, especially when the problem is under-constrained. An extreme example of an under-constrained problem is to start a simulation with a floating plate while the tools are doubly curved. For this purpose, a simulation is performed in which the tools are semi spheres and the plate floats in between. The radius of the punch is $39 \mathrm{~mm}$, the radius of the die is $40 \mathrm{~mm}$. The initial distance between both tools is $27 \mathrm{~mm}$. The plate is $46 \mathrm{~mm} * 46 \mathrm{~mm}$ and the thickness is set to $1 \mathrm{~mm}$. The plate has an offset of $2 \mathrm{~mm}$ in both the $\mathrm{x}$ - and $\mathrm{y}$-direction with respect to the center of the spheres. The simulation setup is given in Figure 10. 

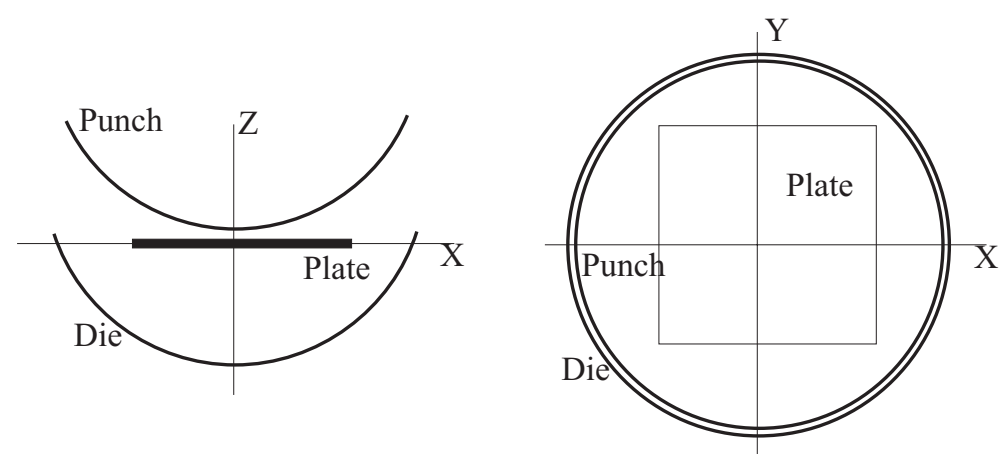

Fig. 10. Schematic of simulation setup.

In practice, the plate will drop due to gravity. Therefore, gravity loads are implemented in finite elements to get a realistic simulation:

$$
\rho g \int_{V} \delta \dot{u} d V=\sum_{\alpha} \delta \dot{u}^{\alpha} \rho g \int_{V} N^{\alpha} d V
$$

The time step is $0.01 \mathrm{~s}$ and the dynamics contributions are switched on. The unbalance criterion is set to 0.02 . In the first 80 steps, the plate drops freely. Then the right upper corner of the plate (see Figure 10) will make contact with the die. Then the plate starts to turn over. Simultaneously, in each incremental step the punch translates $0.2 \mathrm{~mm}$ in negative z-direction. After 20 steps the other plate corners make contact with the die. After a total of 191 steps, the punch and die are fully closed. The deformed plate at different stages during the simulation is shown in Figure 11. Note that this simulation could not be performed when dynamics contributions are not taken into account.

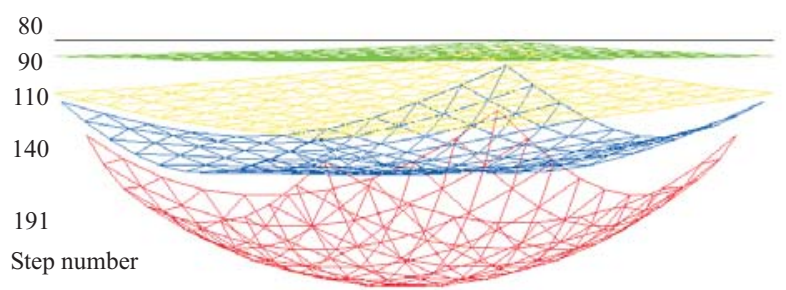

Fig. 11. Deformed plate at different steps of the simulation.

\section{Front Door Panel}

In the last section, the forming of an AUDI-front door panel is discussed. This product served as a benchmark for the Numisheet conference, held in 1999. For more details concerning the geometry of the drawing tools, the reader is referred to [3]. The front view of the drawing tools and the initial blank is given in Figure 12. 
Note that the blankholder is doubly curved which gives rise to instabilities when dynamics contributions are not taken into account. This automotive product will be used to investigate the performance of the improved implicit code with respect to the conventional implicit code.

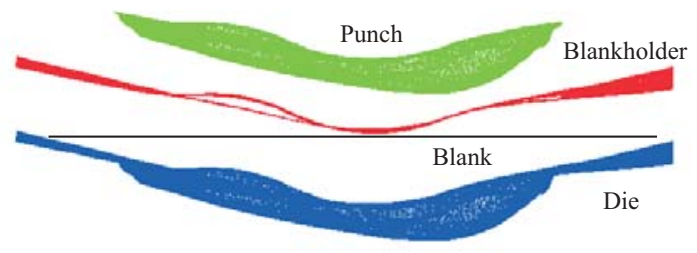

Fig. 12. Drawing tools and initial blank for front door panel.

Two simulations are performed. For both simulations, the dynamics contributions are switched on, gravity loads are applied and automatic refinement is used. The deep drawing velocity is set to $10 \mathrm{~m} / \mathrm{s}$. One simulation is performed in which the Bi-CGSTAB iterative solver with SSOR preconditioning is used (which shows a good performance, see Section 5), and one simulation is performed with a direct solver (Cholesky decomposition).

The discussion of the results is started with the simulation, using the iterative solver. In the first 90 steps, the blankholder is closed. Then the punch moves downwards, until the desired deep drawing depth is reached in step 191. The final deformed mesh is given in Figure 13.

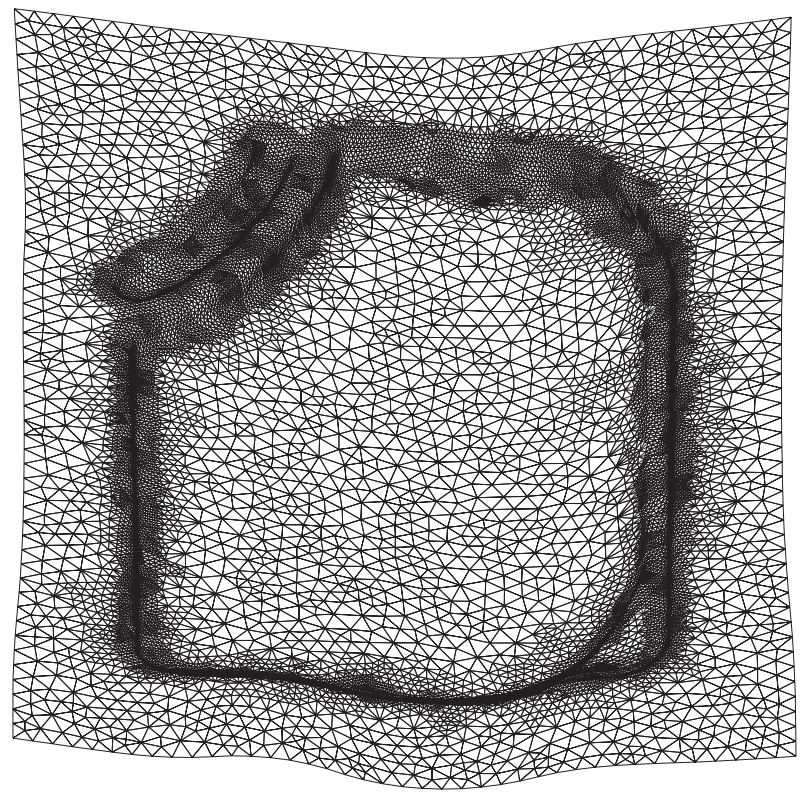

Fig. 13. Final shape of front door panel. 
During deep drawing, the mesh is refined on areas with locally high curvatures [12]. The initial mesh contains 17244 d.o.f., whereas the mesh ends up with 79344 d.o.f. The generation of new elements during the simulation are graphically represented in Figure 14. The total computation time for the entire simulation took 5.5 hours on a HP8000 workstation, which is very fast for an implicit code. This computation time is similar to the computation time of an explicit code for this simulation [3].

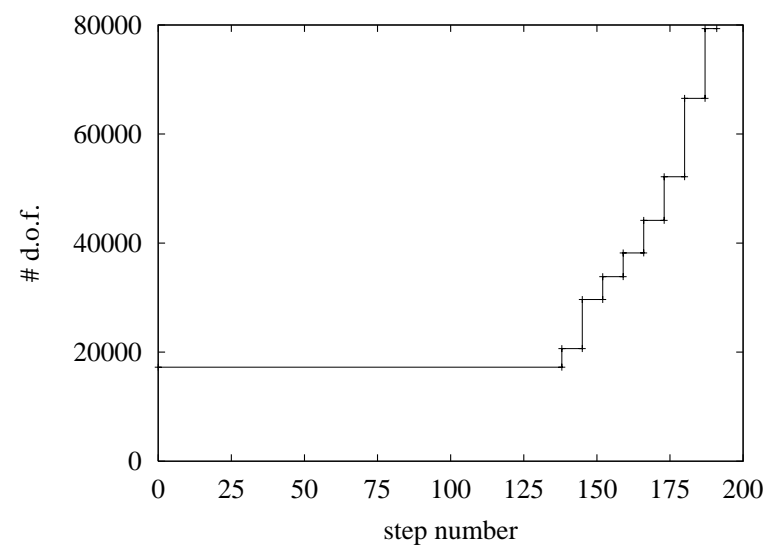

Fig. 14. Increase of d.o.f. during the simulation.

Then, a simulation is started, using a direct solver. Again, in the first 90 steps the blankholder is closed. Then the punch moves downwards, until the mesh contains 36876 d.o.f. At this moment the hardware of the current workstation is not sufficient anymore to proceed the simulation due to insufficient internal memory. Therefore, to make a comparison between the necessary computation time for both solvers, the computation time for one iteration is looked at. The computation time for one iteration strongly depends one the number of d.o.f., see Figure 15. This figure shows the computation time for one iteration for both the simulations with the iterative and direct solver, during the simulation. Besides, the analytical graph for the increase of computation time for the direct solver is added, $n * n_{b}^{2}$ [11], where $n$ is the number of d.o.f. and $n_{b}$ is the bandwidth. In case of a planar uniform mesh, this graph can be represented by $n^{2}$. 


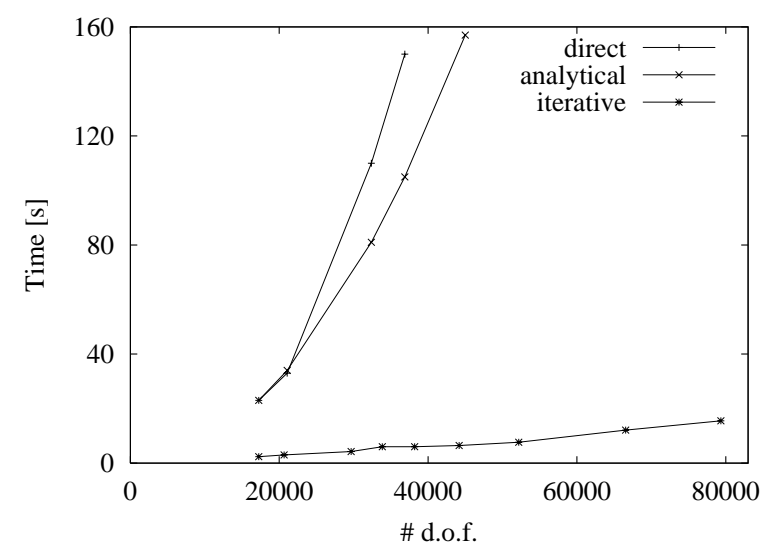

Fig. 15. Computation time for direct and iterative solver.

The figure clearly shows that the iterative solver is significantly faster than the direct solver. For 17244 d.o.f. the iterative solver is 10 times faster than the direct solver while this factor increases up to 25 for 36876 d.o.f. For the direct solver, it is also observed that the computation time increases more than quadratically, which is due to the non-uniformity of the refined mesh. The computation time of the iterative solver increases almost linear. If the analytical approach is taken as a lower bound for the prediction of the computation time for the direct solver in case of 79344 d.o.f., the computation time will increase by at least a factor 40 with respect to the iterative solver. Subsequently it is concluded that the computation time for the iterative solver in combination with dynamics contributions is factors smaller that the computation time when the direct solver is used. However, the total simulation time is not only determined by solving the system matrix, also the creation of the system matrix and the contact search algorithm are time consuming (linear with the number of d.o.f.). Therefore, for this simulation it is stated that when the hardware would be sufficient to perform the entire simulation when using the direct solver, the simulation time would increase by a factor 5-10.

\section{Concluding remarks and recommendation}

The dynamics contributions are successfully implemented in the implicit finite element code DiekA for both the plane strain element (only displacement d.o.f.) and the Mindlin shell element (displacement and rotational d.o.f.). The backward difference scheme and the Newmark integration scheme can be used to calculate the velocities and accelerations, where the backward difference scheme suffers from numerical damping. For use in deep drawing simulations, the backward difference scheme is favorable, since the numerical damping contributes to the stabilization of the simulation. The computation time of a deep drawing simulation with an implicit finite element code is drastically decreased when dynamics contributions in 
combination with an iterative solver are used. For large problems, the computation time to solve the system is decreased by a factor 25 . As a result, the computation time for the entire simulation (including the contact search algorithm and creation of the system matrix) can be decreased by a factor 5-10.

The implementation of dynamics contributions in the implicit finite element code stabilizes the simulation, especially when the problem is under-constrained.

This paper is closed with a final recommendation: at this moment, structural damping is not implemented. Implementation of the structural damping can stabilize the simulation to a higher degree.

\section{References}

[1] K. Mattiasson, L. Bernspang, A. Honecker, E. Schedin, T. Hamman, A. Melander, On the use of explicit time integration in finite element simulation of industrial sheet metal forming processes, in: Numerical simulations of 3D Sheet Forming Processes, VDI-Berichte, VDI Verlag GmBH, Dusseldorf, 1991, pp. 479-498.

[2] J. C. Gelin, B. Liu, C. Labergere, Stable and accurate algorithms for the simulation of deep drawing and tube hydroforming processes, in: E. Onate (Ed.), European Congress on Computational Methods in Applied Sciences and Engineering, CIMNE Publishing, Barcelona, 2000.

[3] Numisheet'99 benchmarks, in: J. C. Gelin, P. Picart (Eds.), Numerical Simulation of 3D Sheet Forming Processes, Volume 2, Burs Edition, Besancon, 1999.

[4] D. Kuhl, M. A. Crisfield, Energy-conserving and decaying algorithms in non-linear structural dynamics, Int. J. Num. Meth. Eng. 45 (1999) 569-599.

[5] J. L. Meriam, L. G. Kraige, Engineering Mechanics Dynamics vol.2 3rd edition, John Wiley and Sons Inc., 1993.

[6] S. S. Rao, Mechanical Vibrations 3rd edition, Addison-Wesley Publishing Company Inc., 1995.

[7] Bruell, Kjaer, Mechanical vibration and shock measurements, K. Larsen and Son A/S, 1984.

[8] J. L. Batoz, P. Lardeur, A discrete shear triangular nine d.o.f. element for the analysis of thick to very thin plates, Int. J. Num. Meth. Eng. 28 (1989) 533-560.

[9] M. A. Eisenberg, L. E. Malvern, On finite element integration in natural coordinates, Int. J. Num. Meth. Eng. 7 (1973) 574-575.

[10] O. C. Ziekiewicz, R. L. Taylor, The Finite Element Method, fourth edition, McGrawHill Book Company, 1987.

[11] A. H. van den Boogaard, A. D. Rietman, J. Huétink, Iterative solvers in forming process simulations, in: J. Huétink, F. P. T. Baaijens (Eds.), Simulation of Materials Processing: Theory, Methods and Applications, A. A. Balkema, Rotterdam, 1998, pp. 219-224. 
[12] T. Meinders, Developments in numerical simulations of the real-life deep drawing process, Ph.D. thesis, University of Twente (2000). 